\title{
Traceless Redox-Annulations of Alicyclic Amines
}

\author{
Dillon R. L. Rickertsen ${ }^{\triangleright a}$ \\ Longle $\mathrm{Ma}^{\diamond \mathrm{b}}$ (D) \\ Anirudra Paul ${ }^{\mathrm{a}}$ \\ Khalil A. Abboudc \\ Daniel Seidel*a (D) \\ ${ }^{a}$ Center for Heterocyclic Compounds, Department of Chemistry, \\ University of Florida, Gainesville, Florida 32611, USA \\ seidel@chem.ufl.edu \\ b Department of Chemistry and Chemical Biology, Rutgers, The \\ State University of New Jersey, Piscataway, New Jersey 08854 , \\ USA \\ ' Center for X-ray Crystallography, Department of Chemistry, \\ University of Florida, Gainesville, Florida 32611, USA \\ $\diamond$ These authors contributed equally
}<smiles>O=Cc1ccccc1C[N+](=O)[O-]</smiles><smiles>c1ccc2c(c1)CCNC2</smiles>
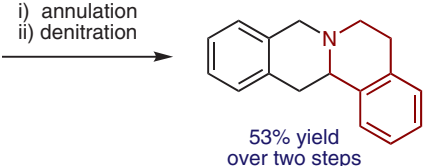

Received: 12.11 .2020

Accepted after revision: 04.12.2020

Published online: 16.12 .2020

DOI: 10.1055/s-0040-1706004; Art ID: so-2020-d0042-op

License terms: $c(0)$

(c) 2020. The Author(s). This is an open access article published by Thieme under the terms of the Creative Commons Attribution-NonDerivative-NonCommercial-License, permitting copying and reproduction so long as the original work is given appropriate credit. Contents may not be used for commercial purposes or adapted, remixed, transformed or built upon. (https://creativecommons.org/licenses/by-nc-nd/4.0/)

Abstract Amines such as 1,2,3,4-tetrahydroisoquinoline undergo redox-neutral annulations with ortho-(nitromethyl)benzaldehyde. Benzoic acid acts as a promoter in these reactions, which involve concurrent amine $\alpha-\mathrm{C}-\mathrm{H}$ bond and $\mathrm{N}-\mathrm{H}$ bond functionalization. Subsequent removal of the nitro group provides access to tetrahydroprotoberberines not accessible via typical redox-annulations. Also reported are decarboxylative annulations of ortho-(nitromethyl)benzaldehyde with proline and pipecolic acid.

Key words $\mathrm{C}-\mathrm{H}$ bond functionalization, redox-neutral, redox-annulation, denitration, decarboxylative annulation

New methods for the $\mathrm{C}-\mathrm{H}$ bond functionalization of amines and their derivatives continue to be developed at a rapid pace. ${ }^{1,2}$ However, few approaches have emerged that are compatible with unprotected secondary amines while at the same time enabling $\alpha-\mathrm{C}-\mathrm{H}$ bond functionalization with concurrent $\mathrm{C}-\mathrm{N}$ bond formation. ${ }^{1 \mathrm{~m}, \mathrm{o}}$ Particularly attractive in this regard are redox-annulations of cyclic amines, which allow for the rapid formation of polycyclic amines from simple starting materials (Scheme 1). Water is the only byproduct in these reactions. Examples of this type of transformation include condensations of amines with ortho-aminobenzaldehydes to provide aminals (Scheme 1a, $\mathrm{X}=\mathrm{NR}),{ }^{3}$ and related, carboxylic-acid-catalyzed transformations involving $\alpha-C-O$ and $\alpha-C-S$ bond formation. ${ }^{4}$ Redoxannulations that achieve $\alpha-C-C$ bond formation with orthotolualdehyde derivatives require the presence of at least one electron-withdrawing group on the ortho-methyl group. ${ }^{5}$ In addition, activation of an ortho-methyl group has been achieved with heteroaryl substrates (Scheme $1 \mathrm{~b})^{6}$ and highly electron-deficient 0 -tolualdehydes (Scheme 1c). ${ }^{7-10}$ Here, we report the first redox-annulations of amines with ortho(nitromethyl)benzaldehydes (Scheme $1 \mathrm{~d}$ ). In these reactions, the nitro group acts as a traceless activator as it can be removed in a subsequent step. The overall strategy represents an attractive new pathway to members and analogues of the tetrahydroprotoberberine family of natural products. $^{11}$

a) Redox-annulation of amines with ortho-substituted benzaldehydes:<smiles>[X]c1ccccc1C=O</smiles><smiles>c1ccc2c(c1)CCNC2</smiles>

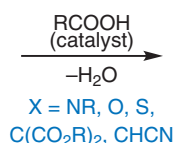<smiles>[X]c1ccccc1CN1CCc2ccccc2C1[N+]#[C-]</smiles>

b) Redox-annulation with 2-alkylquinoline-3-carbaldehydes:<smiles>Cc1nc2ccccc2cc1C=O</smiles><smiles>CC(C)C(C)C(C)C(C)C</smiles><smiles>c1ccc2c(c1)CCN1Cc3cc4ccccc4nc3CC21</smiles>

c) Redox-annulation with electron-deficient ortho-tolualdehydes:<smiles>Cc1cc([N+](=O)[O-])cc([N+](=O)[O-])c1C</smiles>

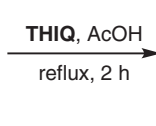

$\stackrel{\mathrm{flux}, 2 \mathrm{~h}}{\longrightarrow}$<smiles>O=[N+]([O-])c1cc2c(c([N+](=O)[O-])c1)CC1c3ccccc3CCN1C2</smiles>

$81 \%$ d) Redox-annulation with removable activating group (this work):<smiles>CC1=C(C)C(P)NCC1</smiles>

i) annulation ii) denitration

Scheme 1 Examples of amine redox-annulations and present work 
ortho-(Nitromethyl)benzaldehyde (1a) $)^{12}$ and 1,2,3,4tetrahydroisoquinoline (THIQ) were selected as the model substrates in the initial evaluation of the proposed redoxannulation. Key optimization experiments are summarized in Table 1. While conditions used in other redox-annulations (reflux in toluene with benzoic acid as a promoter) provided the target product $\mathbf{2 a}$ in substantial amounts, improved results were obtained under microwave conditions. The maximum yield of $76 \%$ was achieved in a reaction that was performed in dichloroethane solvent at $150{ }^{\circ} \mathrm{C}$ for 5 min (entry 4). The reactions exhibited low but variable diastereoselectivities. We suspected that the two diastereomers of 2a may interconvert under the reaction conditions by means of a retro-nitro-Mannich/nitro-Mannich sequence with little thermodynamic preference for either diastereomer. Indeed, while accompanied by some decomposition, exposure of diastereomerically pure $\mathbf{2 a}$ to the reaction conditions led to the recovery of $\mathbf{2} \mathbf{a}$ as a nearly $1: 1$ mixture of diastereomers (Scheme 2).

Table 1 Reaction Development ${ }^{\mathrm{a}}$<smiles>O=Cc1ccccc1C[N+](=O)[O-]</smiles><smiles>c1ccc2c(c1)CCNC2</smiles>

THIQ

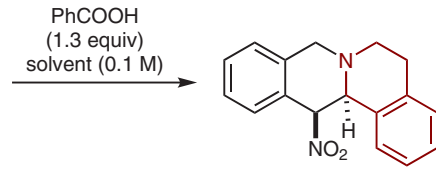

$( \pm)-2 \mathrm{a}$

\begin{tabular}{ccccccc}
\hline Entry & THIQ (equiv) & Solvent & $\mathrm{T}\left({ }^{\circ} \mathrm{C}\right)$ & Time $(\mathrm{min})$ & Yield (\%) & $\mathrm{dr}$ \\
\hline 1 & 1.3 & PhMe & reflux & 60 & 56 & $1: 1$ \\
2 & 1.3 & DCE & reflux & 60 & 58 & $1.3: 1$ \\
$3^{\mathrm{b}}$ & 1.3 & PhMe & 150 & 5 & 61 & $1.1: 1$ \\
$4^{\mathrm{b}}$ & 1.3 & DCE & 150 & 5 & 76 & $1: 1$ \\
$5^{\mathrm{b}}$ & 2.0 & DCE & 150 & 5 & 61 & $1.1: 1$ \\
$6^{\mathrm{b}}$ & 1.3 & DCE & 100 & 5 & 71 & $1.4: 1$ \\
$7^{\mathrm{b}}$ & 1.3 & DCE & 100 & 15 & 75 & $1.2: 1$
\end{tabular}

${ }^{a}$ Reactions were performed on a $0.25 \mathrm{mmol}$ scale. All yields correspond to isolated yields. The $\mathrm{dr}$ was determined by ${ }^{1} \mathrm{H}$ NMR analysis after purification.

b Performed under microwave irradiation.<smiles>O=[N+]([O-])C1c2ccccc2CN2CCc3ccccc3C12</smiles>

$( \pm)-2 a(d r>30: 1)$
$\mathrm{PhCOOH}$ (1.3 equiv)
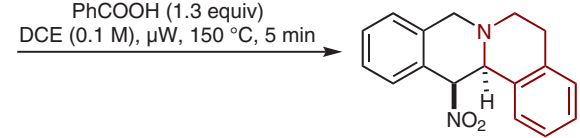

$( \pm)-2 \mathrm{a}(\mathrm{dr}=1.1: 1)$ $60 \%$ recovery DCE $(0.1 \mathrm{M}), \mu \mathrm{W}, 150^{\circ} \mathrm{C}, 5 \mathrm{~min}$

Scheme 2 Equilibration experiment

We then turned our attention to the denitration step (Table 2). Following some optimization, conditions similar to those developed by Carreira and co-workers were found to be efficient in removing the nitro group, ${ }^{13}$ providing product $3 a$ in up to $70 \%$ yield (entry 6 ).
Table 2 Optimization of the Denitration Step ${ }^{\mathrm{a}}$<smiles>O=[N+]([O-])C1c2ccccc2CN2CCc3ccccc3[C@H]12</smiles>

$( \pm)-2 \mathrm{a}$

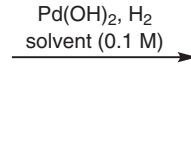

\begin{tabular}{ccclccl}
\hline Entry & Solvent & $\mathrm{H}_{2}$ (atm) & Additive (equiv) & $\mathrm{T}\left({ }^{\circ} \mathrm{C}\right)$ & Time $(\mathrm{h})$ & Yield (\%) \\
\hline 1 & EtOH & 10.2 & - & 85 & $4.5 \mathrm{~h}$ & 57 \\
2 & EtOH & 10.2 & - & $\mathrm{rt}$ & $4.5 \mathrm{~h}$ & trace \\
$3^{\mathrm{b}}$ & $\mathrm{EtOH}$ & 1 & - & 85 & $4.5 \mathrm{~h}$ & trace \\
$4^{\mathrm{b}}$ & $\mathrm{EtOH}$ & 10.2 & - & 85 & $24 \mathrm{~h}$ & 54 \\
5 & $\mathrm{PhMe}$ & 10.2 & - & 85 & $4.5 \mathrm{~h}$ & 54 \\
6 & PhMe & 10.2 & $\mathrm{AcOH}(1.0)$ & 85 & $4.5 \mathrm{~h}$ & 70 \\
7 & PhMe & 10.2 & $\mathrm{AcOH}(2.0)$ & 85 & $4.5 \mathrm{~h}$ & 26 \\
\hline
\end{tabular}

a Reactions were performed on a $0.25 \mathrm{mmol}$ scale. All yields correspond to isolated yields.

${ }^{\mathrm{b}}$ Reaction was performed on a $0.15 \mathrm{mmol}$ scale.

The annulation/denitration sequence was applied to a number of substituted tetrahydroisoquinolines (Scheme 3). Moderate to good yields were achieved in the individual steps with acceptable overall yields. Gratifyingly, 1-aryl tetrahydroisoquinolines with electronically diverse substituents also readily participated in redox-annulations to provide the corresponding sterically congested products as essentially single diastereomers in reasonable yields (Scheme 4). A related tetrahydro- $\beta$-carboline also participated in the reaction but provided the annulation product in significantly lower yield.

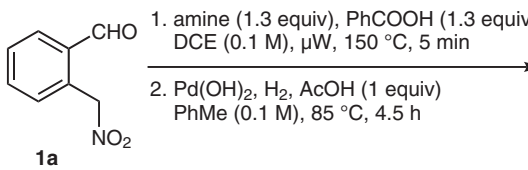

$1 \mathrm{a}$

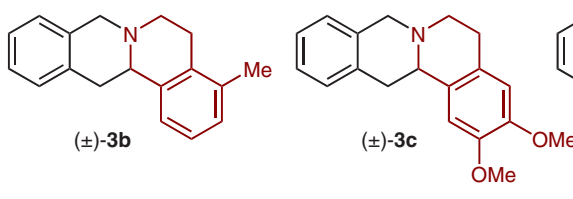

annulation: $73 \%$ denitration: $65 \%$ overall: $47 \%$

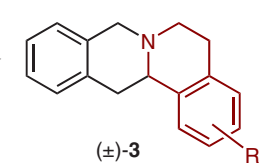

$( \pm)-3$<smiles>c1ccc2c(c1)CC1c3cc4c(cc3CCN1C2)OCO4</smiles>

annulation: $73 \%$ denitration: $52 \%$ overall: $38 \%$
Scheme 3 Evaluation of substituted tetrahydroisoquinolines

Unfortunately, the products shown in Scheme 4 were not amenable to denitration under the reaction conditions employed above. However, removal of the nitro group was readily achieved with tributyltin hydride (Scheme 5). ${ }^{14}$

Despite significant experimentation, less activated amines such as pyrrolidine and piperidine did not participate in redox-annulations with ortho-(nitromethyl)benzaldehyde (1a). However, as has been shown in a number of 


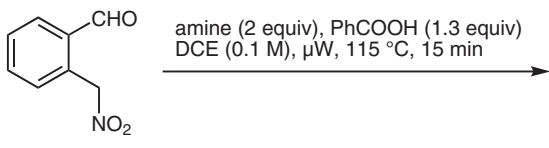

$1 a$<smiles>O=[N+]([O-])C1c2ccccc2CN2CCc3ccccc3[C@]12C1CCCC1</smiles>

( \pm )-2e, $70 \%$ $\mathrm{dr}>20: 1$<smiles>O=[N+]([Te])[C@H]1c2ccccc2CN2CCc3ccccc3[C@]12c1ccc(Br)cc1</smiles>
$( \pm)-2 h, 71 \%$
$d r>20: 1$

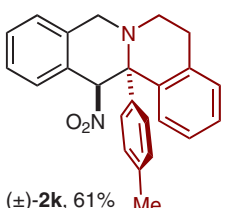
$( \pm)-2 k, 61 \%$ Me
$\mathrm{dr}>20: 1$<smiles>Cc1ccccc1[C@]12c3ccccc3CCN1Cc1ccccc1C2[N+](=O)[O-]</smiles>
$( \pm)-2 f, 64 \%$ $\mathrm{dr}>20: 1$

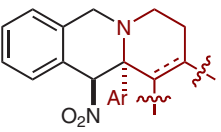

$( \pm)-2$<smiles>O=[N+]([Te])[C@H]1c2ccccc2CN2CCc3ccccc3[C@]12c1ccccc1Cl</smiles>
$\mathrm{dr}>20: 1$ $( \pm)-2 \mathbf{i}, 69$
$\mathrm{dr}>20: 1$<smiles>Cc1cccc2c1CCN1Cc3ccccc3C([N+](=O)[O-])[C@@]21c1ccccc1</smiles>
( \pm )-2I, $60 \%$ $( \pm)-21,60 \%$
$d r>20: 1$<smiles>[O][N+](=O)C1c2ccccc2CN2CCc3ccccc3[C@]12c1ccccc1C(F)(F)F</smiles>

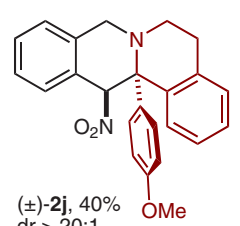
$\mathrm{dr}>20: 1$

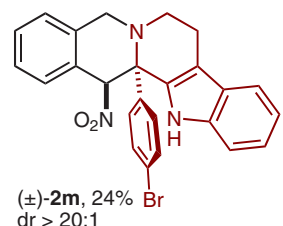

Scheme 4 Formation of sterically congested tetrahydroprotoberberine analogues

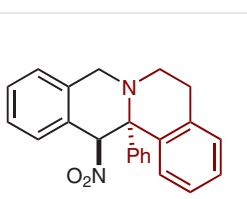

$( \pm)-2 e$

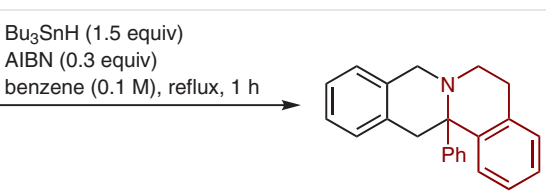

( \pm )-3e, $72 \%$
Scheme 5 Denitration of a sterically congested annulation product

related reactions, ${ }^{15,16}$ the corresponding decarboxylative reactions in which proline and pipecolic acid are used in place of pyrrolidine and piperidine provided annulation products in good yields (Scheme 6). Denitration under Carreira conditions was also successful.

In conclusion, we have achieved the first traceless redox-annulations of amines using a substrate with an activating nitro group that can be subsequently removed. This strategy provides access to products that are not readily available by using conventional synthetic approaches.

Starting materials, reagents, and solvents were purchased from commercial sources and used as received unless stated otherwise. 1,2,3,4Tetrahydroisoquinoline was freshly distilled prior to use. L-Proline, L/D-pipecolic acid, 2,2'-(diazene-1,2-diyl)bis(2-methylpropanenitrile), and tributyltin hydride were used as received. HPLC grade 1,2-dichloroethane (DCE) was purchased from Sigma-Aldrich and was used without further purification. Purification of reaction prod-
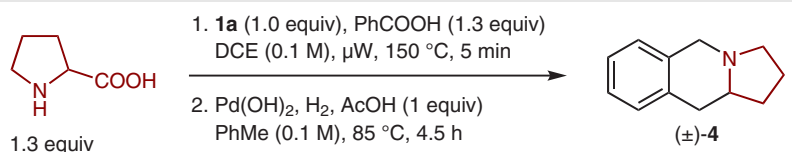

$( \pm)-4$

annulation: $76 \%$ denitration: $69 \%$ overall: $52 \%$

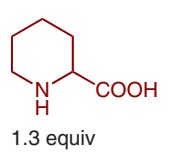

1. 1a (1.0 equiv), $\mathrm{PhCOOH}$ (1.3 equiv) DCE $(0.1 \mathrm{M}), \mu \mathrm{W}, 150^{\circ} \mathrm{C}, 5 \mathrm{~min}$

2. $\mathrm{Pd}(\mathrm{OH})_{2}, \mathrm{H}_{2}, \mathrm{AcOH}$ (1 equiv) PhMe $(0.1 \mathrm{M}), 85^{\circ} \mathrm{C}, 4.5 \mathrm{~h}$

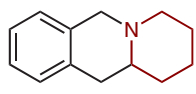

$( \pm)-5$

annulation: $65 \%$ denitration: $73 \%$ overall: $47 \%$

Scheme 6 Decarboxylative annulation/denitration

ucts was carried out by flash column chromatography using Sorbent Technologies Standard Grade silica gel (60 A,, 230-400 mesh). Analytical thin-layer chromatography was performed on EM Reagent 0.25 $\mathrm{mm}$ silica gel 60 F254 plates. Visualization was accomplished with UV light and Dragendorff-Munier stains, followed by heating. ${ }^{1} \mathrm{H}$ NMR spectra were recorded with a Bruker $400 \mathrm{MHz}$ or Bruker $600 \mathrm{MHz}$ instrument and chemical shifts are reported in ppm using the solvent as an internal standard $\left(\mathrm{CDCl}_{3}\right.$ at $\left.7.26 \mathrm{ppm}\right)$. Data are reported as app $=$ apparent, $\mathrm{s}=$ singlet, $\mathrm{d}=$ doublet, $\mathrm{t}=$ triplet, $\mathrm{q}=$ quartet, $\mathrm{m}=$ multiplet, comp = complex, br = broad; coupling constant(s) in Hz. Protondecoupled carbon nuclear magnetic resonance spectra $\left({ }^{13} \mathrm{C} \mathrm{NMR}\right)$ spectra were recorded with a Bruker $400 \mathrm{MHz}$ or Bruker $600 \mathrm{MHz}$ instrument and chemical shifts are reported in ppm using the solvent as an internal standard $\left(\mathrm{CDCl}_{3}\right.$ at $\left.77.16 \mathrm{ppm}\right)$. Diastereomeric ratios of the products were determined by ${ }^{1} \mathrm{H}$ NMR analysis of the purified products. Accurate mass data (ESI) was obtained with Agilent 1260 Infinity II LC/MSD using MassWorks 5.0 from CERNO bioscience. ${ }^{17}$ Reactions under microwave irradiation were conducted with a Biotage Initiator+, SW version: 4.1.4 build 11991 .

1-Phenyl-1,2,3,4-tetrahydroisoquinoline, ${ }^{18 \mathrm{a}} \quad$ 1-(4-fluorophenyl)1,2,3,4-tetrahydroisoquinoline, ${ }^{18 \mathrm{~b}}$ 1-(4-chlorophenyl)-1,2,3,4-tetrahydroisoquinoline, ${ }^{18 \mathrm{~b}} 1$-(4-bromophenyl)-1,2,3,4-tetrahydroisoquinoline, ${ }^{18 \mathrm{c}}$ 1-(4-(trifluoromethyl)phenyl)-1,2,3,4-tetrahydroisoquinoline, ${ }^{18 \mathrm{~d}} 1$-(4-methoxyphenyl)-1,2,3,4-tetrahydroisoquinoline, ${ }^{18 \mathrm{~b}} 1$-( $p$ tolyl)-1,2,3,4-tetrahydroisoquinoline, ${ }^{18 \mathrm{~b}} \quad 1$-( $m$-tolyl)-1,2,3,4-tetrahydroisoquinoline, ${ }^{18 e}$ 1-(4-bromophenyl)-2,3,4,9-tetrahydro-1H-pyrido[3,4-b]indole, ${ }^{18 f}$ 6,7-dimethoxy-1,2,3,4-tetrahydroisoquinoline, ${ }^{18 g}$ 5,6,7,8-tetrahydro-[1,3]dioxolo[4,5-g]isoquinoline, ${ }^{18 \mathrm{~h}}$ 5-methyl1,2,3,4-tetrahydroisoquinoline, ${ }^{18 \mathrm{i}}$ and 2-(nitromethyl)benzaldehyde ${ }^{18 \mathrm{j}}$ were prepared according to reported procedures and their published characterization data matched our own in all respects.

\section{3-Nitro-5,8,13,13a-tetrahydro-6H-isoquinolino[3,2-a]isoquino-} line (2a)

2-(Nitromethyl)benzaldehyde (1a) ( $41.3 \mathrm{mg}, 0.25 \mathrm{mmol}, 1$ equiv), 1,2,3,4-tetrahydroisoquinoline ( $41.5 \mu \mathrm{L}, 0.33 \mathrm{mmol}, 1.3$ equiv), and benzoic acid ( $40.3 \mathrm{mg}, 0.33 \mathrm{mmol}, 1.3$ equiv) were added to a microwave vial charged with a stir bar. Dichloroethane $(2.5 \mathrm{~mL})$ was added and the microwave vial was sealed. The vial was stirred until complete dissolution of the solids and then placed in the microwave, followed by heating for 5 minutes at $150{ }^{\circ} \mathrm{C}$ with the instrument set to low absorption. The reaction mixture was neutralized with sat. $\mathrm{NaHCO}_{3}(15 \mathrm{~mL})$ and extracted with EtOAc $(3 \times 15 \mathrm{~mL})$. The combined organic layers were dried over $\mathrm{Na}_{2} \mathrm{SO}_{4}$. The solvent was removed 
under reduced pressure and the crude residue was purified by silica gel chromatography using hexanes containing EtOAc (0-15\%), yielding $2 \mathbf{a}$ as a mixture of diastereomers with a dr of $1: 1$.

Yield: 76\% (53.3 mg); brown oil; $R_{f}=0.16$ (hexanes/EtOAc 90:10 v/v). ${ }^{1} \mathrm{H}$ NMR $\left(600 \mathrm{MHz}, \mathrm{CDCl}_{3}\right): \delta=7.47(\mathrm{dd}, J=7.7,1.3 \mathrm{~Hz}, 0.5 \mathrm{H}), 7.43-$ 7.35 (comp, $1 \mathrm{H}$ ), 7.34-7.10 (comp, $6 \mathrm{H}$ ), 7.04-6.96 (m, $0.5 \mathrm{H}$ ), 6.17 (d, $J=3.3 \mathrm{~Hz}, 0.5 \mathrm{H}), 5.90(\mathrm{~d}, J=8.6 \mathrm{~Hz}, 0.5 \mathrm{H}), 4.76(\mathrm{~d}, J=8.6 \mathrm{~Hz}, 0.5 \mathrm{H})$, $4.38(\mathrm{dd}, J=15.8,1.3 \mathrm{~Hz}, 0.5 \mathrm{H}), 4.26(\mathrm{~d}, J=15.3 \mathrm{~Hz}, 0.5 \mathrm{H}), 4.20(\mathrm{~d}, J=$ $3.3 \mathrm{~Hz}, 0.5 \mathrm{H}), 3.96$ (d, $J=15.8 \mathrm{~Hz}, 0.5 \mathrm{H}), 3.79(\mathrm{~d}, J=15.3 \mathrm{~Hz}, 0.5 \mathrm{H})$, 3.33-3.19 (comp, $1 \mathrm{H}$ ), 3.08-2.96 (comp, $2 \mathrm{H}$ ), 2.92-2.85 (m, $0.5 \mathrm{H}$ ), $2.77-2.69(\mathrm{~m}, 0.5 \mathrm{H})$.

${ }^{13} \mathrm{C}$ NMR $\left(151 \mathrm{MHz}, \mathrm{CDCl}_{3}\right): \delta=136.4,136.4,134.7,134.7,134.1$, 130.0, 129.6, 129.6, 129.5, 129.3, 128.6, 127.8, 127.7, 127.4, 127.3, $127.2,127.0,127.0,126.9,126.6,126.3,126.0,125.7,90.1,87.0,63.3$, $62.1,57.8,56.7,50.8,48.0,29.3,29.2$.

HRMS (ESI): $m / z[\mathrm{M}+\mathrm{H}]^{+}$calcd for $\mathrm{C}_{17} \mathrm{H}_{17} \mathrm{~N}_{2} \mathrm{O}_{2}: 281.1285$; found: 281.1655. Spectral Accuracy: 98.8\%.

\section{General Procedure A}

2-(Nitromethyl)benzaldehyde (1a) $(82.6 \mathrm{mg}, 0.5 \mathrm{mmol}, 1$ equiv), amine ( $0.65 \mathrm{mmol}, 1.3$ equiv), and benzoic acid $(79.4 \mathrm{mg}, 0.65 \mathrm{mmol}$, 1.3 equiv) were added to a microwave vial charged with a stir bar. Dichloroethane $(5.0 \mathrm{~mL})$ was added and the microwave vial was sealed. The vial was stirred until complete dissolution of the solids and placed in the microwave, followed by heating for 5 minutes at $150{ }^{\circ} \mathrm{C}$ with the instrument set to low absorption. The reaction mixture was neutralized with sat. $\mathrm{NaHCO}_{3}(20 \mathrm{~mL})$ and extracted with EtOAc $(3 \times$ $20 \mathrm{~mL}$ ). The combined organic layers were dried over $\mathrm{Na}_{2} \mathrm{SO}_{4}$. The solvent was removed under reduced pressure and the crude residue was purified by silica gel chromatography. The product was used directly in the next step.

\section{General Procedure B}

The annulation product obtained according to General Procedure A was added to a reaction vial charged with acetic acid (1.0 equiv) and a stir bar. Toluene $(5.0 \mathrm{~mL})$ was added followed by $20 \%$ wt. $\mathrm{Pd}(\mathrm{OH})_{2} / \mathrm{C}$ $(66.7 \mathrm{mg})$. The reaction vial was placed in a bomb and back filled with $\mathrm{H}_{2}(5 \times) . \mathrm{H}_{2}$ was added to the bomb until the internal pressure reached 150 PSI. The reaction mixture was heated at $85{ }^{\circ} \mathrm{C}$ for 4.5 hours. The reaction mixture was then allowed to cool to r.t., followed by removal of the solvent under reduced pressure. The crude mixture was purified by silica gel chromatography followed by treatment with sat. $\mathrm{NaHCO}_{3}(15 \mathrm{~mL})$ and extraction with EtOAc $(3 \times 15 \mathrm{~mL})$. The combined organic layers were dried over $\mathrm{Na}_{2} \mathrm{SO}_{4}$. The solvent was removed under reduced pressure yielding the final product.

\section{General Procedure C}

2-(Nitromethyl)benzaldehyde (1a) ( $41.3 \mathrm{mg}, 0.25 \mathrm{mmol}, 1$ equiv), amine ( $0.50 \mathrm{mmol}, 2.0$ equiv), and benzoic acid $(40.3 \mathrm{mg}, 0.33 \mathrm{mmol}$, 1.3 equiv) were added to a microwave vial charged with a stir bar. Dichloroethane $(2.5 \mathrm{~mL})$ was added and the microwave vial was sealed. The vial was stirred until complete dissolution of the solids and placed in the microwave, followed by heating for 15 minutes at $115^{\circ} \mathrm{C}$ with the microwave set to low absorption. The reaction mixture was neutralized with sat. $\mathrm{NaHCO}_{3}(15 \mathrm{~mL})$ and extracted with EtOAc $(3 \times$ $15 \mathrm{~mL}$ ). The combined organic layers were dried over $\mathrm{Na}_{2} \mathrm{SO}_{4}$. The solvent was removed under reduced pressure and the crude residue was purified by silica gel chromatography.

\section{3-Nitro-13a-phenyl-5,8,13,13a-tetrahydro-6H-isoquinolino-} [3,2-a]isoquinoline (2e)

By following General Procedure C, compound ( \pm )-2e was obtained from aldehyde $1 \mathbf{a}$ (41.3 mg, $0.25 \mathrm{mmol}$, 1equiv) and 1-phenyl-1,2,3,4tetrahydroisoquinoline ( $104 . \mathrm{g} \mathrm{mg}, 0.5 \mathrm{mmol}, 2.0$ equiv). Hexanes containing EtOAc (0-10\%) was used as the eluent for silica gel chromatography.

Yield: 70\% (62.4 mg) and a > 20:1 diastereomeric ratio; white solid; $R_{f}=0.13$ (hexanes $/$ EtOAc $95: 5 \mathrm{v} / \mathrm{v}$ ).

${ }^{1} \mathrm{H}$ NMR $\left(600 \mathrm{MHz}, \mathrm{CDCl}_{3}\right): \delta=7.58(\mathrm{dd}, J=7.6,1.5 \mathrm{~Hz}, 1 \mathrm{H}), 7.39-7.34$ (comp, $2 \mathrm{H}$ ), 7.25-7.16 (comp, $3 \mathrm{H}$ ), 7.15-7.09 (comp, $4 \mathrm{H}$ ), 7.03 (dd, $J=8.0,1.2 \mathrm{~Hz}, 1 \mathrm{H}), 6.83-6.78(\mathrm{comp}, 2 \mathrm{H}), 6.59(\mathrm{~s}, 1 \mathrm{H}), 3.93(\mathrm{~d}, J=$ $16.3 \mathrm{~Hz}, 1 \mathrm{H}$ ), 3.39-3.26 (comp, $3 \mathrm{H}$ ), 3.07-3.00 (m, $1 \mathrm{H}$ ), 2.91-2.84 $(\mathrm{m}, 1 \mathrm{H})$.

${ }^{13} \mathrm{C}$ NMR $\left(151 \mathrm{MHz}, \mathrm{CDCl}_{3}\right): \delta=139.6,136.9,136.8,136.0,129.6$, $129.2,128.9,128.5,128.4,128.3,127.8,127.6,127.5,127.3,126.8$, $126.2,91.5,65.8,52.3,45.6,29.5$.

HRMS (ESI): $m / z[M+H]^{+}$calcd for $\mathrm{C}_{23} \mathrm{H}_{21} \mathrm{~N}_{2} \mathrm{O}_{2}$ : 357.1598; found: 357.1589. Spectral Accuracy: 97.3\%.

13a-(4-Fluorophenyl)-13-nitro-5,8,13,13a-tetrahydro-6H-isoquinolino[3,2-a]isoquinoline (2f)

By following General Procedure $C$, compound ( \pm )-2f was obtained from aldehyde $\mathbf{1 a}$ ( $41.3 \mathrm{mg}, 0.25 \mathrm{mmol}, 1$ equiv) and 1-(4-fluorophenyl)-1,2,3,4-tetrahydroisoquinoline (113.g $\mathrm{mg}, 0.5 \mathrm{mmol}, 2.0$ equiv). Hexanes containing EtOAc (0-10\%) was used as the eluent for silica gel chromatography.

Yield: 64\% (59.9 mg) and a > 20:1 diastereomeric ratio; off-white solid; $R_{f}=0.30$ (hexanes/EtOAc $90: 10 \mathrm{v} / \mathrm{v}$ ).

${ }^{1} \mathrm{H}$ NMR $\left(600 \mathrm{MHz}, \mathrm{CDCl}_{3}\right): \delta=7.56(\mathrm{dd}, J=7.7,1.4 \mathrm{~Hz}, 1 \mathrm{H}), 7.38$ (app td, $J=7.5,1.5 \mathrm{~Hz}, 1 \mathrm{H}$ ), 7.34 (app td, $J=7.5,1.4 \mathrm{~Hz}, 1 \mathrm{H}$ ), 7.23-7.11 (comp, $4 \mathrm{H}$ ), 7.00 (dd, $J=7.9,1.3 \mathrm{~Hz}, 1 \mathrm{H}$ ), 6.82 (app t, $J=8.7 \mathrm{~Hz}, 2 \mathrm{H}$ ), 6.79-6.74 (comp, $2 \mathrm{H}$ ), 6.53 (s, $1 \mathrm{H}$ ), $3.94(\mathrm{~d}, J=16.3 \mathrm{~Hz}, 1 \mathrm{H}), 3.37-$ 3.27 (comp, $2 \mathrm{H}$ ), 3.21 (app td, $J=11.6,3.3 \mathrm{~Hz}, 1 \mathrm{H}$ ), 3.03 (ddd, $J=11.8$, 6.0, $1.9 \mathrm{~Hz}, 1 \mathrm{H}$ ), 2.85 (app dt, $J=15.6,2.7 \mathrm{~Hz}, 1 \mathrm{H}$ ).

${ }^{13} \mathrm{C} \mathrm{NMR}\left(151 \mathrm{MHz}, \mathrm{CDCl}_{3}\right): \delta=161.8\left(\mathrm{~d}, J_{\mathrm{C}-\mathrm{F}}=247.8 \mathrm{~Hz}\right), 136.7,136.0$, $135.4\left(\mathrm{~d}, J_{\mathrm{C}-\mathrm{F}}=3.2 \mathrm{~Hz}\right), 130.2\left(\mathrm{~d}, J_{\mathrm{C}-\mathrm{F}}=7.8 \mathrm{~Hz}\right), 129.8,129.0,128.9$, $128.4,128.2,127.6,127.5,126.9,126.3,114.7\left(\mathrm{~d}, J_{\mathrm{C}-\mathrm{F}}=21.0 \mathrm{~Hz}\right), 91.5$, $65.4,52.2,45.5,29.5$.

HRMS (ESI): $m / z[\mathrm{M}+\mathrm{H}]^{+}$calcd for $\mathrm{C}_{23} \mathrm{H}_{20} \mathrm{FN}_{2} \mathrm{O}_{2}$ : 375.1503; found: 375.1379. Spectral Accuracy: $97.4 \%$.

\section{3a-(4-Chlorophenyl)-13-nitro-5,8,13,13a-tetrahydro-6H-iso- quinolino[3,2-a] isoquinoline (2g)}

By following General Procedure C, compound ( \pm )-2g was obtained from aldehyde $1 \mathrm{a}$ ( $41.3 \mathrm{mg}, 0.25 \mathrm{mmol}, 1$ equiv) and 1-(4-chlorophenyl)-1,2,3,4-tetrahydroisoquinoline ( $121.9 \mathrm{mg}, 0.5 \mathrm{mmol}, 2.0$ equiv). Hexanes containing EtOAc (0-15\%) was used as the eluent for silica gel chromatography.

Yield: 66\% (64.5 mg) and > 20:1 diastereomeric ratio; off-white solid; $R_{f}=0.52$ (hexanes/EtOAc 80:20 v/v).

${ }^{1} \mathrm{H} \mathrm{NMR}\left(600 \mathrm{MHz}, \mathrm{CDCl}_{3}\right): \delta=7.56(\mathrm{dd}, J=7.6,1.4 \mathrm{~Hz}, 1 \mathrm{H}), 7.39-7.33$ (comp, $2 \mathrm{H}$ ), 7.29-7.11 (comp, $6 \mathrm{H}$ ), 7.06-6.96 (m, $1 \mathrm{H}$ ), 6.76-6.71 (comp, $2 \mathrm{H}$ ), $6.52(\mathrm{~s}, 1 \mathrm{H}$ ), 3.95 (d, J = 16.3 Hz, $1 \mathrm{H}$ ), 3.38-3.27 (comp, 2 H), 3.22 (app td, $J=11.6,3.2 \mathrm{~Hz}, 1 \mathrm{H}$ ), 3.05 (dd, $J=12.0,5.8 \mathrm{~Hz}, 1 \mathrm{H}$ ), $2.85(\mathrm{~d}, J=15.5 \mathrm{~Hz}, 1 \mathrm{H})$. 
${ }^{13} \mathrm{C}$ NMR $\left(151 \mathrm{MHz}, \mathrm{CDCl}_{3}\right): \delta=138.1,136.6,136.4,136.0,133.6$, $129.8,129.8,129.0,128.8,128.4,128.2,128.0,127.6,127.6,126.8$, 126.3, 91.3, 65.5, 52.2, 45.6, 29.4 .

HRMS (ESI): $m / z[\mathrm{M}+\mathrm{H}]^{+}$calcd for $\mathrm{C}_{23} \mathrm{H}_{20} \mathrm{ClN}_{2} \mathrm{O}_{2}: 391.1208$; found: 391.1429. Spectral Accuracy: 97.2\%.

\section{3a-(4-Bromophenyl)-13-nitro-5,8,13,13a-tetrahydro-6H-iso- quinolino[3,2-a]isoquinoline $(\mathbf{2 h})$}

By following General Procedure C, compound $( \pm)-\mathbf{2 h}$ was obtained from aldehyde 1a (41.3 mg, $0.25 \mathrm{mmol}, 1$ equiv) and 1-(4-bromophenyl)-1,2,3,4-tetrahydroisoquinoline ( $144.1 \mathrm{mg}, 0.5 \mathrm{mmol}, 2.0$ equiv). Hexanes containing EtOAc (0-10\%) was used as the eluent for silica gel chromatography.

Yield: 71\% (77.3 mg) and > 20:1 diastereomeric ratio; off-white solid; $R_{f}=0.27$ (hexanes/EtOAc 90:10 v/v).

${ }^{1} \mathrm{H}$ NMR $\left(600 \mathrm{MHz}, \mathrm{CDCl}_{3}\right): \delta=7.58(\mathrm{dd}, J=7.7,1.4 \mathrm{~Hz}, 1 \mathrm{H}), 7.42-7.35$ (comp, 2 H), 7.33-7.25 (comp, 2 H), 7.25-7.08 (comp, 4 H), 7.08-7.00 (m, $1 \mathrm{H}$ ), 6.72-6.67 (comp, $2 \mathrm{H}), 6.54(\mathrm{~s}, 1 \mathrm{H}), 3.97$ (d, $J=16.3 \mathrm{~Hz}, 1 \mathrm{H}$ ), 3.41-3.29 (comp, $2 \mathrm{H}$ ), 3.25 (app td, $J=11.6,3.2 \mathrm{~Hz}, 1 \mathrm{H}$ ), 3.07 (ddd, $J=$ 12.0, 6.0, $2.0 \mathrm{~Hz}, 1 \mathrm{H}$ ), 2.87 (app dt, $J=15.4,2.6 \mathrm{~Hz}, 1 \mathrm{H}$ ).

${ }^{13} \mathrm{C}$ NMR $\left(151 \mathrm{MHz}, \mathrm{CDCl}_{3}\right): \delta=138.6,136.6,136.3,136.0,131.0$, 130.1, 129.8, 129.0, 128.8, 128.4, 128.2, 127.6, 127.6, 126.8, 126.3, $121.8,91.2,65.5,52.2,45.5,29.4$

HRMS (ESI): $m / z[\mathrm{M}+\mathrm{H}]^{+}$calcd for $\mathrm{C}_{23} \mathrm{H}_{20} \mathrm{BrN}_{2} \mathrm{O}_{2}$ : 435.0703; found: 435.0610. Spectral Accuracy: 98.1\%.

\section{3-Nitro-13a-(4-(trifluoromethyl)phenyl)-5,8,13,13a-tetrahydro- 6H-isoquinolino[3,2-a] isoquinoline (2i)}

By following General Procedure C, compound ( \pm )-2i was obtained from aldehyde 1a (41.3 mg, $0.25 \mathrm{mmol}, 1$ equiv) and 1-(4-(trifluoromethyl)phenyl)-1,2,3,4-tetrahydroisoquinoline (138.6 mg, $0.5 \mathrm{mmol}$, 2.0 equiv). Hexanes containing EtOAc (0-10\%) was used as the eluent for silica gel chromatography.

Yield: $69 \%$ (73.2 mg) and > 20:1 diastereomeric ratio; off-white solid; $R_{f}=0.30$ (hexanes $/$ EtOAc $90: 10 \mathrm{v} / \mathrm{v}$ ).

${ }^{1} \mathrm{H}$ NMR $\left(600 \mathrm{MHz}, \mathrm{CDCl}_{3}\right): \delta=7.58(\mathrm{dd}, J=7.6,1.5 \mathrm{~Hz}, 1 \mathrm{H}), 7.43-7.32$ (comp, 4 H), 7.24-7.17 (comp, $2 \mathrm{H}$ ), 7.14 (app ddt, $J=6.5,4.6,2.1 \mathrm{~Hz}, 2$ $\mathrm{H}), 6.99(\mathrm{dd}, J=7.9,1.2 \mathrm{~Hz}, 1 \mathrm{H}), 6.94(\mathrm{~d}, J=8.3 \mathrm{~Hz}, 2 \mathrm{H}), 6.57(\mathrm{~s}, 1 \mathrm{H})$, $3.97(\mathrm{~d}, J=16.4 \mathrm{~Hz}, 1 \mathrm{H}), 3.40-3.30$ (comp, $2 \mathrm{H}$ ), 3.26 (app td, $J=11.5$, $3.0 \mathrm{~Hz}, 1 \mathrm{H}), 3.18-3.05$ ( $\mathrm{m}, 1 \mathrm{H}), 2.89$ (dd, $J=15.7,2.9 \mathrm{~Hz}, 1 \mathrm{H}$ ).

${ }^{13} \mathrm{C}$ NMR $\left(151 \mathrm{MHz}, \mathrm{CDCl}_{3}\right): \delta=143.7,136.5,136.0,129.9,129.7$ (q, $\left.J_{\mathrm{C}-\mathrm{F}}=32.6 \mathrm{~Hz}\right), 129.2,128.8,128.7,128.5,128.2,127.7,126.9,126.4$, $124.8\left(\mathrm{q}, J_{\mathrm{C}-\mathrm{F}}=3.8 \mathrm{~Hz}\right), 123.9\left(\mathrm{q}, J_{\mathrm{C}-\mathrm{F}}=272.4 \mathrm{~Hz}\right), 91.1,65.6,52.2,45.6$, 29.4 .

HRMS (ESI): $m / z$ [M $+\mathrm{H}]^{+}$calcd for $\mathrm{C}_{24} \mathrm{H}_{20} \mathrm{~F}_{3} \mathrm{~N}_{2} \mathrm{O}_{2}$ : 425.1471; found: 425.1820. Spectral Accuracy: 97.5\%.

13a-(4-Methoxyphenyl)-13-nitro-5,8,13,13a-tetrahydro-6H-isoquinolino[3,2-a]isoquinoline $(2 \mathrm{j})$

By following General Procedure C, compound $( \pm)-\mathbf{2} \mathbf{j}$ was obtained from aldehyde 1a (41.3 mg, $0.25 \mathrm{mmol}, 1$ equiv) and 1-(4-methoxyphenyl)-1,2,3,4-tetrahydroisoquinoline (119.7 mg, $0.5 \mathrm{mmol}, 2.0$ equiv). Hexanes containing EtOAc (0-10\%) was used as the eluent for silica gel chromatography.

Yield: 40\% (38.6 mg) and > 20:1 diastereomeric ratio; off-white solid; $R_{f}=0.19$ (hexanes/EtOAc 90:10 v/v).
${ }^{1} \mathrm{H}$ NMR $\left(600 \mathrm{MHz}, \mathrm{CDCl}_{3}\right): \delta=7.60-7.54(\mathrm{~m}, 1 \mathrm{H}), 7.39-7.32$ (comp, 2 H), 7.21-7.09 (comp, $4 \mathrm{H}$ ), 7.07-6.97 (m, $1 \mathrm{H}$ ), 6.73-6.68 (comp, $2 \mathrm{H}$ ), 6.68-6.62 (comp, $2 \mathrm{H}), 6.54(\mathrm{~s}, 1 \mathrm{H}), 3.91(\mathrm{~d}, J=16.1 \mathrm{~Hz}, 1 \mathrm{H}), 3.71(\mathrm{~s}, 3$ H), 3.39-3.27 (comp, $2 \mathrm{H}$ ), 3.23 (app td, $J=11.6,3.2 \mathrm{~Hz}, 1 \mathrm{H}$ ), 3.00 (ddd, $J=11.7,5.9,1.9 \mathrm{~Hz}, 1 \mathrm{H}), 2.84(\mathrm{app} \mathrm{dt}, J=15.4,2.6 \mathrm{~Hz}, 1 \mathrm{H}$ ).

${ }^{13} \mathrm{C}$ NMR $\left(151 \mathrm{MHz}, \mathrm{CDCl}_{3}\right): \delta=158.7,137.2,136.9,136.0,131.6$, $129.8,129.6,129.2,128.9,128.4,128.3,127.5,127.3,126.8,126.1$, 113.0, 91.7, 65.5, 55.2, 52.3, 45.5, 29.6.

HRMS (ESI): $m / z[\mathrm{M}+\mathrm{H}]^{+}$calcd for $\mathrm{C}_{24} \mathrm{H}_{23} \mathrm{~N}_{2} \mathrm{O}_{3}$ : 387.1703; found: 387.1899. Spectral Accuracy: $98.8 \%$.

\section{3-Nitro-13a-( $p$-tolyl)-5,8,13,13a-tetrahydro-6H-isoquinolino-} [3,2-a]isoquinoline (2k)

By following General Procedure $C$, compound $( \pm)-2 \mathbf{k}$ was obtained from aldehyde 1a (41.3 mg, $0.25 \mathrm{mmol}, 1$ equiv) and 1 -( $p$-tolyl)1,2,3,4-tetrahydroisoquinoline ( $111.7 \mathrm{mg}, 0.5 \mathrm{mmol}, 2.0$ equiv). Hexanes containing EtOAc (0-10\%) was used as the eluent for silica gel chromatography.

Yield: $61 \%$ (56.5 mg) and > 20:1 diastereomeric ratio; off-white solid; $R_{f}=0.32$ (hexanes/EtOAc 90:10 v/v)

${ }^{1} \mathrm{H}$ NMR $\left(600 \mathrm{MHz}, \mathrm{CDCl}_{3}\right): \delta=7.57(\mathrm{~d}, J=7.9,1.2 \mathrm{~Hz}, 1 \mathrm{H}), 7.40-7.31$ (comp, $2 \mathrm{H}$ ), 7.24-7.15 (comp, $2 \mathrm{H}$ ), 7.12 (ddd, $J=9.5,7.1,1.9 \mathrm{~Hz}, 2 \mathrm{H}$ ), 7.03 (d, $J=7.9,1.2 \mathrm{~Hz}, 1 \mathrm{H}$ ), 6.94 (d, $J=8.2 \mathrm{~Hz}, 2 \mathrm{H}$ ), 6.70-6.65 (comp, $2 \mathrm{H}), 6.57(\mathrm{~s}, 1 \mathrm{H}), 3.91(\mathrm{~d}, J=16.2 \mathrm{~Hz}, 1 \mathrm{H}), 3.39-3.24$ (comp, $3 \mathrm{H}$ ), 3.05-2.99 (m, $1 \mathrm{H}), 2.85$ (dd, $J=15.3,3.0 \mathrm{~Hz}, 1 \mathrm{H}), 2.24(\mathrm{~s}, 3 \mathrm{H})$.

${ }^{13} \mathrm{C}$ NMR $\left(151 \mathrm{MHz}, \mathrm{CDCl}_{3}\right): \delta=137.3,137.1,136.9,136.5,136.0$, $129.5,129.3,128.9,128.5,128.5,128.4,128.3,127.4,127.2,126.8$, $126.1,91.62,65.7,52.3,45.56,29.6,21.0$.

HRMS (ESI): $m / z$ [M + H] $]^{+}$calcd for $\mathrm{C}_{24} \mathrm{H}_{23} \mathrm{~N}_{2} \mathrm{O}_{2}$ : 371.1759; found: 371.1935. Spectral Accuracy: 97.5\%.

\section{3-Nitro-13a-(m-tolyl)-5,8,13,13a-tetrahydro-6H-isoquinolino- [3,2-a]isoquinoline (21)}

By following General Procedure C, compound ( \pm )-2l was obtained from aldehyde 1a $(41.3 \mathrm{mg}, 0.25 \mathrm{mmol}, 1$ equiv) and 1 -( $m$-tolyl)1,2,3,4-tetrahydroisoquinoline ( $111.7 \mathrm{mg}, 0.5 \mathrm{mmol}, 2.0$ equiv). Hexanes containing EtOAc (0-10\%) was used as the eluent for silica gel chromatography.

Yield: $60 \%$ (55.6 mg) and > 20:1 diastereomeric ratio; off-white solid; $R_{f}=0.28$ (hexanes/EtOAc 90:10 v/v).

${ }^{1} \mathrm{H}$ NMR $\left(600 \mathrm{MHz}, \mathrm{CDCl}_{3}\right): \delta=7.57(\mathrm{dd}, J=7.6,1.5 \mathrm{~Hz}, 1 \mathrm{H}), 7.38-7.32$ (comp, $2 \mathrm{H}$ ), 7.23-7.16 (comp, $2 \mathrm{H}$ ), 7.12 (app ddt, $J=6.4,4.5,2.2 \mathrm{~Hz}, 2$ H), 7.07-6.96 (comp, $3 \mathrm{H}$ ), 6.66-6.54 (comp, $3 \mathrm{H}$ ), 3.92 (d, $J=16.2 \mathrm{~Hz}$, $1 \mathrm{H}), 3.39$ (d, $J=16.2 \mathrm{~Hz}, 1 \mathrm{H}$ ), 3.35-3.27 (comp, $2 \mathrm{H}$ ), 3.09-3.01 (m, 1 $\mathrm{H}), 2.92-2.83(\mathrm{~m}, 1 \mathrm{H}), 2.15(\mathrm{~s}, 3 \mathrm{H})$.

${ }^{13} \mathrm{C}$ NMR $\left(151 \mathrm{MHz}, \mathrm{CDCl}_{3}\right): \delta=139.6,137.3,136.9,136.8,135.9$, $129.4,129.4,129.2,128.8,128.5,128.3,128.3,127.5,127.3,127.2$, $126.7,126.0,125.3,91.5,65.7,52.3,45.5,29.5,21.8$.

HRMS (ESI): $m / z$ [M + H] calcd for $\mathrm{C}_{24} \mathrm{H}_{23} \mathrm{~N}_{2} \mathrm{O}_{2}$ : 371.1754; found: 371.2009. Spectral Accuracy: $98.2 \%$.

13b-(4-Bromophenyl)-14-nitro-5,7,8,13,13b,14-hexahydroindolo[2',3':3,4]pyrido[1,2-b]isoquinoline ( $2 \mathrm{~m})$

2-(Nitromethyl)benzaldehyde ( $82.6 \mathrm{mg}, 0.5 \mathrm{mmol}, 1$ equiv), 1-(4-bromophenyl)-2,3,4,9-tetrahydro- $1 H$-pyrido[3,4-b]indole (327.2 mg, 1.0 mmol, 2.0 equiv), and benzoic acid ( $79.4 \mathrm{mg}, 0.65 \mathrm{mmol}, 1.3$ equiv) were added to a microwave vial charged with a stir bar. Dichloroethane $(5.0 \mathrm{~mL})$ was added and the microwave vial was sealed. The vial was stirred and placed in the microwave, followed by heating for 
15 minutes at $115{ }^{\circ} \mathrm{C}$ with the microwave set to low absorption. The reaction mixture was neutralized with sat. $\mathrm{NaHCO}_{3}(20 \mathrm{~mL})$ and extracted with EtOAc $(3 \times 20 \mathrm{~mL})$. The combined organic layers were dried over $\mathrm{Na}_{2} \mathrm{SO}_{4}$. The solvent was removed under reduced pressure and the crude residue purified by silica gel chromatography using hexanes containing EtOAc (0-15\%) as the eluent, yielding $\mathbf{2 m}$.

Yield: $24 \%$ (56.9 mg) and > 20:1 diastereomeric ratio; pale-green solid; $R_{f}=0.40$ (hexanes/EtOAc 80:20 v/v).

${ }^{1} \mathrm{H}$ NMR $\left(600 \mathrm{MHz}, \mathrm{CDCl}_{3}\right): \delta=7.83(\mathrm{~s}, 1 \mathrm{H}), 7.59(\mathrm{~d}, J=7.7 \mathrm{~Hz}, 1 \mathrm{H})$, 7.49-7.41 (comp, $2 \mathrm{H}$ ), 7.38 (app td, $J=7.5,1.3 \mathrm{~Hz}, 1 \mathrm{H}$ ), 7.32-7.26 (comp, $4 \mathrm{H}$ ), 7.24-7.19 (comp, $2 \mathrm{H}$ ), 6.73 (d, $J=8.3 \mathrm{~Hz}, 2 \mathrm{H}$ ), 6.51 (s, 1 H), $4.08(\mathrm{~d}, J=16.3 \mathrm{~Hz}, 1 \mathrm{H}), 3.48$ (d, $J=16.3 \mathrm{~Hz}, 1 \mathrm{H}), 3.22$ (app td, $J=$ $12.7,11.9,3.9 \mathrm{~Hz}, 1 \mathrm{H}$ ), 3.13 (app ddt, $J=16.4,10.7,4.4 \mathrm{~Hz}, 2 \mathrm{H}$ ), 2.98$2.91(\mathrm{~m}, 1 \mathrm{H})$.

${ }^{13} \mathrm{C}$ NMR $\left(151 \mathrm{MHz}, \mathrm{CDCl}_{3}\right): \delta=137.0,131.5,130.9,130.0,129.9$, 128.6, 128.1, 127.8, 127.0, 126.4, 122.9, 119.9, 118.9, 113.4, 111.5, $90.0,63.8,51.5,46.7,21.3$.

HRMS (ESI): $m / z[\mathrm{M}+\mathrm{H}]^{+}$calcd for $\mathrm{C}_{25} \mathrm{H}_{21} \mathrm{BrN}_{3} \mathrm{O}_{2}: 474.0812$; found: 474.0616. Spectral Accuracy: 97.7\%.

\section{$\mathbf{5 , 8 , 1 3 , 1 3 a - T e t r a h y d r o - 6} \boldsymbol{H}$-isoquinolino[3,2-a]isoquinoline (3a)}

By following General Procedures A and B, compound ( \pm )-3a was obtained from aldehyde $\mathbf{1 a}(82.6 \mathrm{mg}, 0.5 \mathrm{mmol}, 1$ equiv) and 1,2,3,4-tetrahydroisoquinoline ( $81.7 \mu \mathrm{L}, 0.65 \mathrm{mmol}, 1.3$ equiv). Hexanes containing EtOAc (0-20\%) was used as the eluent for silica gel chromatography. Characterization data for 3a match literature reports in all respects. ${ }^{19 a, 19 b}$

Yield: 53\% (62.4 mg) over two steps; yellow solid; $R_{f}=0.39$ (hexanes/EtOAc 70:30 v/v).

${ }^{1} \mathrm{H}$ NMR $\left(400 \mathrm{MHz}, \mathrm{CDCl}_{3}\right): \delta=7.30(\mathrm{~d}, J=7.0 \mathrm{~Hz}, 1 \mathrm{H}), 7.26-7.14$ (comp, $6 \mathrm{H}$ ), 7.10 (dd, $J=6.5,2.7 \mathrm{~Hz}, 1 \mathrm{H}), 4.06(\mathrm{~d}, J=14.9 \mathrm{~Hz}, 1 \mathrm{H}$ ), 3.85-3.67 (comp, $2 \mathrm{H}$ ), 3.48-3.36 (m, $1 \mathrm{H}$ ), 3.32-3.15 (comp, $2 \mathrm{H}$ ), 2.96 (ddd, $J=16.3,11.3,1.8 \mathrm{~Hz}, 1 \mathrm{H}$ ), $2.85-2.75$ (m, $1 \mathrm{H}$ ), 2.72-2.62 (m, $1 \mathrm{H})$.

${ }^{13} \mathrm{C}$ NMR $\left(101 \mathrm{MHz}, \mathrm{CDCl}_{3}\right): \delta=136.0,134.6,134.6,134.5,129.0$, 128.9, 126.4, 126.3, 126.3, 126.2, 126.0, 125.6, 60.0, 58.7, 51.3, 36.8, 29.6.

HRMS (ESI): $m / z[M+H]^{+}$calcd for $\mathrm{C}_{17} \mathrm{H}_{18} \mathrm{~N}$ : 236.1434; found: 236.1526. Spectral Accuracy: 98.6\%.

\section{4-Methyl-5,8,13,13a-tetrahydro-6H-isoquinolino[3,2-a]isoquino- line (3b)}

By following General Procedures A and B, compound ( \pm )-3b was obtained from aldehyde $1 \mathrm{a}(82.6 \mathrm{mg}, 0.5 \mathrm{mmol}, 1$ equiv) and 5-methyl1,2,3,4-tetrahydroisoquinoline ( $95.7 \mathrm{mg}, 0.65 \mathrm{mmol}, 1.3$ equiv). Hexanes containing EtOAc (0-10\%) was used as the eluent for silica gel chromatography.

Yield: $47 \%$ (58.6 mg) over two steps; white solid; $R_{f}=0.25$ (hexanes/EtOAc 90:10 v/v).

${ }^{1} \mathrm{H}$ NMR (600 MHz, $\mathrm{CDCl}_{3}$ ): $\delta=7.28-7.17$ (comp, $5 \mathrm{H}$ ), 7.13-7.10 (comp, $2 \mathrm{H}$ ), 4.09 (d, $J=14.9 \mathrm{~Hz}, 1 \mathrm{H}$ ), 3.87-3.69 (comp, $2 \mathrm{H}$ ), 3.42 (dd, $J=16.3,4.1 \mathrm{~Hz}, 1 \mathrm{H}$ ), 3.27 (ddd, $J=11.5,5.8,2.2 \mathrm{~Hz}, 1 \mathrm{H}$ ), 3.10-2.88 (comp, $2 \mathrm{H}$ ), 2.77 (app dt, $J=16.5,2.9 \mathrm{~Hz}, 1 \mathrm{H}$ ), 2.67 (app td, $J=11.4$, $3.8 \mathrm{~Hz}, 1 \mathrm{H}), 2.31$ (s, $3 \mathrm{H})$.

${ }^{13} \mathrm{C}$ NMR (151 MHz, $\left.\mathrm{CDCl}_{3}\right): \delta=138.0,136.3,134.6,134.4,133.2$, 128.8, 127.6, 126.4, 126.2, 125.9, 125.9, 123.3, 60.1, 58.8, 51.2, 36.9, $27.1,19.4$
HRMS (ESI): $m / z[M+H]^{+}$calcd for $\mathrm{C}_{18} \mathrm{H}_{20} \mathrm{~N}$ : 250.1590; found: 250.1705. Spectral Accuracy: 99.0\%.

\section{2,3-Dimethoxy-5,8,13,13a-tetrahydro-6H-isoquinolino[3,2-a]iso- quinoline (3c)}

By following General Procedures A and B, compound ( \pm )-3c was obtained from aldehyde $\mathbf{1 a}(82.6 \mathrm{mg}, 0.5 \mathrm{mmol}, 1$ equiv) and 6,7-dimethoxy-1,2,3,4-tetrahydroisoquinoline (125.6 mg, $0.65 \mathrm{mmol}, 1.3$ equiv). Hexanes containing EtOAc (0-40\%) was used as the eluent for silica gel chromatography. Characterization data for $\mathbf{3 c}$ match a literature report in all respects. ${ }^{19 \mathrm{c}}$

Yield: $34 \%$ (50.2 mg) over two steps; white solid; $R_{f}=0.14$ (hexanes/EtOAc 75:25 v/v).

${ }^{1} \mathrm{H}$ NMR $\left(600 \mathrm{MHz}, \mathrm{CDCl}_{3}\right): \delta=7.20-7.12(\mathrm{comp}, 3 \mathrm{H}), 7.11-7.06(\mathrm{~m}, 1$ $\mathrm{H}), 6.75(\mathrm{~s}, 1 \mathrm{H}), 6.62(\mathrm{~s}, 1 \mathrm{H}), 4.04(\mathrm{~d}, J=14.9 \mathrm{~Hz}, 1 \mathrm{H}), 3.90(\mathrm{~s}, 3 \mathrm{H})$, $3.87(\mathrm{~s}, 3 \mathrm{H}), 3.78-3.73(\mathrm{~m}, 1 \mathrm{H}), 3.70-3.62(\mathrm{~m}, 1 \mathrm{H}), 3.34$ (dd, $J=16.2$, $3.9 \mathrm{~Hz}, 1 \mathrm{H}$ ), 3.20-3.12 (comp, $2 \mathrm{H}$ ), 2.98-2.87 (m, $1 \mathrm{H}$ ), 2.73-2.60 (comp, $2 \mathrm{H}$ )

${ }^{13} \mathrm{C}$ NMR $\left(151 \mathrm{MHz}, \mathrm{CDCl}_{3}\right): \delta=147.6,147.6,134.4,129.7,128.8$, 126.7, 126.4, 126.2, 126.0, 111.4, 108.6, 59.6, 58.6, 56.2, 55.9, 51.4, $36.8,29.0$.

HRMS (ESI): $m / z[\mathrm{M}+\mathrm{H}]^{+}$calcd for $\mathrm{C}_{19} \mathrm{H}_{22} \mathrm{NO}_{2}$ : 296.1645; found: 296.1739. Spectral Accuracy: 98.6\%.

\section{$5,8,13,13 a-T e t r a h y d r o-6 H-[1,3]$ dioxolo[4,5-g]isoquinolino- [3,2-a]isoquinoline (3d)}

By following General Procedures A and B, compound ( \pm )-3d was obtained from aldehyde $1 \mathrm{a}(82.6 \mathrm{mg}, 0.5 \mathrm{mmol}, 1$ equiv) and 5,6,7,8-tetrahydro[1,3]dioxolo[4,5-g]isoquinoline (115.2 mg, $0.65 \mathrm{mmol}, 1.3$ equiv). Hexanes containing EtOAc (0-20\%) was used as the eluent for silica gel chromatography. Characterization data for 3d match a literature report in all respects. ${ }^{19 b}$

Yield: $38 \%\left(53.1 \mathrm{mg}\right.$ ) over two steps; white solid; $R_{f}=0.28$ (hexanes/EtOAc 75:25 v/v).

${ }^{1} \mathrm{H}$ NMR (600 MHz, $\mathrm{CDCl}_{3}$ ): $\delta=7.21-7.13$ (comp, $3 \mathrm{H}$ ), 7.11-7.05 (m, 1 $\mathrm{H}), 6.76(\mathrm{~s}, 1 \mathrm{H}), 6.60(\mathrm{~s}, 1 \mathrm{H}), 5.92-5.91$ (comp, $2 \mathrm{H}), 4.03$ (d, $J=14.9$ $\mathrm{Hz}, 1 \mathrm{H}), 3.75(\mathrm{~d}, J=14.9 \mathrm{~Hz}, 1 \mathrm{H}), 3.62(\mathrm{dd}, J=11.2,4.0 \mathrm{~Hz}, 1 \mathrm{H}), 3.29$ (dd, $J=16.2,4.0 \mathrm{~Hz}, 1 \mathrm{H}$ ), 3.18-3.09 (comp, $2 \mathrm{H}$ ), 2.95-2.87 (m, $1 \mathrm{H}$ ), 2.71-2.58 (comp, $2 \mathrm{H}$ ).

${ }^{13} \mathrm{C}$ NMR $\left(151 \mathrm{MHz}, \mathrm{CDCl}_{3}\right): \delta=146.3,146.1,134.4,134.3,130.8$, $128.8,127.8,126.4,126.2,126.0,108.5,105.6,100.9,60.0,58.6,51.4$, 36.9, 29.6 .

HRMS (ESI): $m / z[\mathrm{M}+\mathrm{H}]^{+}$calcd for $\mathrm{C}_{18} \mathrm{H}_{18} \mathrm{NO}_{2}$ : 280.1332; found: 280.1565. Spectral Accuracy: 99.1\%.

13a-Phenyl-5,8,13,13a-tetrahydro-6H-isoquinolino[3,2-a]isoquinoline (3e)

Compound ( \pm )-2e (71.3 mg, $0.20 \mathrm{mmol}$. 1.0 equiv), and AIBN (9.9 mg, $0.06 \mathrm{mmol}, 0.3$ equiv) was added to benzene $(2.0 \mathrm{~mL})$ and stirred until complete dissolution. Tributyltin hydride $(80.9 \mu \mathrm{L}, 0.3 \mathrm{mmol}, 1.5$ equiv) was then added and the reaction mixture was heated under reflux for 1 hour. The reaction mixture was extracted with $1 \mathrm{M} \mathrm{HCl}(3 \times$ $10 \mathrm{~mL}$ ) and the combined aqueous layers were basified with $1 \mathrm{M}$ $\mathrm{NaOH}$. The aqueous layer was back extracted with EtOAc $(3 \times 15 \mathrm{~mL})$ and the combined organic layers were dried over $\mathrm{Na}_{2} \mathrm{SO}_{4}$. The solvent was removed under reduced pressure and the crude residue was purified by silica gel chromatography using hexanes containing EtOAc ( 0 $5 \%$ ) as the eluent yielding $3 \mathbf{e}$.

Yield: 72\% (44.8 mg); white solid; $R_{f}=0.33$ (hexanes/EtOAc 95:5 v/v). 
${ }^{1} \mathrm{H}$ NMR $\left(600 \mathrm{MHz}, \mathrm{CDCl}_{3}\right): \delta=7.25-7.13$ (comp, $10 \mathrm{H}$ ), 7.06 (app td, $J=7.4,1.7 \mathrm{~Hz}, 1 \mathrm{H}), 6.98(\mathrm{~d}, J=7.5 \mathrm{~Hz}, 1 \mathrm{H}), 6.78(\mathrm{dd}, J=7.9,1.3 \mathrm{~Hz}, 1$ H), 3.71-3.55 (comp, $3 \mathrm{H}), 3.44(\mathrm{~d}, J=17.5 \mathrm{~Hz}, 1 \mathrm{H}), 3.28-3.23(\mathrm{~m}, 1$ H), 3.17 (ddd, $J=11.8,8.3,4.7 \mathrm{~Hz}, 1 \mathrm{H}$ ), 3.09 (app dt, $J=11.9,5.3 \mathrm{~Hz}, 1$ H), 3.02 (app dt, $J=15.8,4.8 \mathrm{~Hz}, 1 \mathrm{H}$ ).

${ }^{13} \mathrm{C}$ NMR $\left(151 \mathrm{MHz}, \mathrm{CDCl}_{3}\right): \delta=134.5,134.2,133.4,129.8,128.9$, $128.9,128.4,128.2,127.9,127.8,126.9,126.5,126.4,126.0,126.0$, $126.0,62.5,53.5,46.5,36.2,29.9$.

HRMS (ESI): $m / z[M+H]^{+}$calcd for $\mathrm{C}_{23} \mathrm{H}_{22} \mathrm{~N}$ : 312.1747; found: 312.1787. Spectral Accuracy: $97.4 \%$.

\section{1,2,3,5,10,10a-Hexahydropyrrolo[1,2-b]isoquinoline (4)}

By following General Procedures A and B, compound ( \pm )-4 was obtained from aldehyde 1a ( $82.6 \mathrm{mg}, 0.5 \mathrm{mmol}, 1$ equiv) and L-proline (74.8 mg, $0.65 \mathrm{mmol}, 1.3$ equiv). Dichloromethane containing $\mathrm{MeOH}$ $(0-10 \%)$ was used as the eluent for silica gel chromatography. Characterization data for $\mathbf{4}$ match a literature report in all respects. ${ }^{19}$

Yield: $52 \%$ (45.0 mg) over two steps; colorless oil; $R_{f}=0.13\left(\mathrm{CH}_{2} \mathrm{Cl}_{2} /\right.$ $\mathrm{MeOH} 96: 4 \mathrm{v} / \mathrm{v})$

${ }^{1} \mathrm{H}$ NMR $\left(600 \mathrm{MHz}, \mathrm{CDCl}_{3}\right): \delta=7.13-7.10(\mathrm{comp}, 3 \mathrm{H}), 7.11-7.05(\mathrm{~m}, 1$ H), $4.16(\mathrm{~d}, J=14.6 \mathrm{~Hz}, 1 \mathrm{H}), 3.47(\mathrm{~d}, J=14.6 \mathrm{~Hz}, 1 \mathrm{H}), 3.30(\mathrm{app} \mathrm{td}, J=$ 8.7, $2.5 \mathrm{~Hz}, 1 \mathrm{H}$ ), 3.01 (dd, $J=15.9,3.9 \mathrm{~Hz}, 1 \mathrm{H}), 2.78-2.71(\mathrm{~m}, 1 \mathrm{H})$, 2.42-2.36 (m, $1 \mathrm{H}$ ), 2.31 (app q, $J=8.8 \mathrm{~Hz}, 1 \mathrm{H}$ ), 2.12 (dddd, $J=12.3$, 9.8, 6.8, $4.2 \mathrm{~Hz}, 1 \mathrm{H}$ ), 1.95 (app ddtd, $J=12.7,11.2,8.6,4.2 \mathrm{~Hz}, 1 \mathrm{H}$ ), $1.89-1.79(\mathrm{~m}, 1 \mathrm{H}), 1.58$ (dddd, $J=12.3,11.3,9.8,6.8 \mathrm{~Hz}, 1 \mathrm{H}$ ).

${ }^{13} \mathrm{C}$ NMR $\left(151 \mathrm{MHz}, \mathrm{CDCl}_{3}\right): \delta=135.0,134.9,129.1,126.7,126.3$, $125.8,60.8,55.9,54.8,36.0,31.1,21.7$

HRMS (ESI): $m / z[M+H]^{+}$calcd for $\mathrm{C}_{12} \mathrm{H}_{16} \mathrm{~N}$ : 174.1277; found: 174.1276. Spectral Accuracy: $99.4 \%$.

\section{1,3,4,6,11,11a-Hexahydro-2H-pyrido[1,2-b]isoquinoline (5)}

By following General Procedures A and B, compound ( \pm ) $\mathbf{5}$ was obtained from aldehyde $1 \mathrm{a}(82.6 \mathrm{mg}, 0.5 \mathrm{mmol}, 1$ equiv) and L/Dpipecolic acid ( $84.0 \mathrm{mg}, 0.65 \mathrm{mmol}, 1.3$ equiv). Dichloromethane containing $\mathrm{MeOH}(0-4 \%)$ was used as the eluent for silica gel chromatography. Characterization data for $\mathbf{5}$ match a literature report in all respects. ${ }^{19 \mathrm{~d}}$

Yield: $47 \%$ (44.0 mg) over two steps; white solid; $R_{f}=0.18$ in EtOAc. ${ }^{1} \mathrm{H}$ NMR $\left(600 \mathrm{MHz}, \mathrm{CDCl}_{3}\right.$ ): $\delta=7.12-7.08$ (comp, $2 \mathrm{H}$ ), 7.06-7.03 (m, 1 H), 7.02-6.98 (m, $1 \mathrm{H}), 3.86(\mathrm{~d}, J=15.1 \mathrm{~Hz}, 1 \mathrm{H}), 3.39$ (d, $J=15.1 \mathrm{~Hz}, 1$ H), 3.12-3.05 (m, 1 H), 2.90-2.62 (comp, 2 H), 2.25 (app tt, $J=10.2$, $4.2 \mathrm{~Hz}, 1 \mathrm{H}$ ), 2.12 (app td, $J=11.4,4.2 \mathrm{~Hz}, 1 \mathrm{H}$ ), 1.88-1.76 (comp, $2 \mathrm{H}$ ), 1.76-1.67 (comp, $2 \mathrm{H}$ ), 1.42-1.32 (comp, $2 \mathrm{H}$ ).

${ }^{13} \mathrm{C}$ NMR $\left(151 \mathrm{MHz}, \mathrm{CDCl}_{3}\right): \delta=134.3,134.0,128.1,126.2,126.0$, $125.6,58.4,58.4,56.2,36.8,33.7,25.9,24.3$.

HRMS (ESI): $m / z[M+H]^{+}$calcd for $\mathrm{C}_{13} \mathrm{H}_{18} \mathrm{~N}$ : 188.1434; found: 188.1383. Spectral Accuracy: $99.2 \%$.

\section{Funding Information}

Financial support from the NIH-NIGMS (Grant R01GM101389) is gratefully acknowledged. We further acknowledge the National Science Foundation (grant \# 1828064 to K.A.A.) and the University of Florida for funding the purchase of the X-ray equipment.

\section{Acknowledgment}

We thank Dr. Ion Ghiviriga (University of Florida) for assistance with NMR experiments.

\section{Supporting Information}

Supporting information for this article is available online at https://doi.org/10.1055/s-0040-1706004.

\section{References}

(1) Selected recent reviews on amine $\mathrm{C}-\mathrm{H}$ functionalization, including redox-neutral approaches: (a) Campos, K. R. Chem. Soc. Rev. 2007, 36, 1069. (b) Jazzar, R.; Hitce, J.; Renaudat, A.; Sofack-Kreutzer, J.; Baudoin, O. Chem. Eur. J. 2010, 16, 2654. (c) Yeung, C. S.; Dong, V. M. Chem. Rev. 2011, 111, 1215. (d) Mitchell, E. A.; Peschiulli, A.; Lefevre, N.; Meerpoel, L.; Maes, B. U. W. Chem. Eur. J. 2012, 18, 10092. (e) Jones, K. M.; Klussmann, M. Synlett 2012, 23, 159. (f) Peng, B.; Maulide, N. Chem. Eur. J. 2013, 19, 13274. (g) Girard, S. A.; Knauber, T.; Li, C.J. Angew. Chem. Int. Ed. 2014, 53, 74. (h) Haibach, M. C.; Seidel, D. Angew. Chem. Int. Ed. 2014, 53, 5010. (i) Wang, L.; Xiao, J. Adv. Synth. Catal. 2014, 356, 1137. (j) Vo, C.-V. T.; Bode, J. W. J. Org. Chem. 2014, 79, 2809. (k) Seidel, D. Org. Chem. Front. 2014, 1, 426. (l) Qin, Y.; Lv, J.; Luo, S. Tetrahedron Lett. 2014, 55, 551. (m) Seidel, D. Acc. Chem. Res. 2015, 48, 317. (n) Beatty, J. W.; Stephenson, C. R. J. Acc. Chem. Res. 2015, 48, 1474. (o) Mahato, S.; Jana, C. K. Chem. Rec. 2016, 16, 1477. (p) Qin, Y.; Zhu, L.; Luo, S. Chem. Rev. 2017, 117, 9433. (q) Cheng, M.-X.; Yang, S.-D. Synlett 2017, 28, 159. (r) Chu, J. C. K.; Rovis, T. Angew. Chem. Int. Ed. 2018, 57, 62. (s) Gonnard, L.; Guérinot, A.; Cossy, J. Tetrahedron 2019, 75, 145. (t) Liu, S.; Zhao, Z.; Wang, Y. Chem. Eur. J. 2019, 25, 2423. (u) Antermite, D.; Bull, J. A. Synthesis 2019, 51, 3171. (v) Trowbridge, A.; Walton, S. M.; Gaunt, M. J. Chem. Rev. 2020, 120, 2613.

(2) Recent examples of mechanistically diverse amine $\mathrm{C}-\mathrm{H}$ bond functionalization reactions: (a) Zhao, Z.; Luo, Y.; Liu, S.; Zhang, L.; Feng, L.; Wang, Y. Angew. Chem. Int. Ed. 2018, 57, 3792. (b) Wang, F.; Rafiee, M.; Stahl, S. S. Angew. Chem. Int. Ed. 2018, 57, 6686. (c) Greßies, S.; Klauck, F. J. R.; Kim, J. H.; Daniliuc, C. G.; Glorius, F. Angew. Chem. Int. Ed. 2018, 57, 9950. (d) Griffiths, R. J.; Kong, W. C.; Richards, S. A.; Burley, G. A.; Willis, M. C.; Talbot, E. P. A. Chem. Sci. 2018, 9, 2295. (e) Idiris, F. I. M.; Majeste, C. E.; Craven, G. B.; Jones, C. R. Chem. Sci. 2018, 9, 2873. (f) Li, S.-S.; Lv, X.; Ren, D.; Shao, C.-L.; Liu, Q.; Xiao, J. Chem. Sci. 2018, 9, 8253. (g) Maier, A. F. G.; Tussing, S.; Zhu, H.; Wicker, G.; Tzvetkova, P.; Flörke, U.; Daniliuc, C. G.; Grimme, S.; Paradies, J. Chem. Eur. J. 2018, 24, 16287. (h) Mori, K.; Isogai, R.; Kamei, Y.; Yamanaka, M.; Akiyama, T. J. Am. Chem. Soc. 2018, 140, 6203. (i) Shang, M.; Chan, J. Z.; Cao, M.; Chang, Y.; Wang, Q.; Cook, B.; Torker, S.; Wasa, M. J. Am. Chem. Soc. 2018, 140, 10593. (j) Lennox, A. J. J.; Goes, S. L.; Webster, M. P.; Koolman, H. F.; Djuric, S. W.; Stahl, S. S. J. Am. Chem. Soc. 2018, 140, 11227. (k) Zhang, J.; Park, S.; Chang, S. J. Am. Chem. Soc. 2018, 140, 13209. (l) Nauth, A. M.; Schechtel, E.; Dören, R.; Tremel, W.; Opatz, T. J. Am. Chem. Soc. 2018, 140, 14169. (m) Jiang, H.-J.; Zhong, X.-M.; Yu, J.; Zhang, Y.; Zhang, X.; Wu, Y.-D.; Gong, L.-Z. Angew. Chem. Int. Ed. 2019, 58, 1803. (n) Ashley, M. A.; Yamauchi, C.; Chu, J. C. K.; Otsuka, S.; Yorimitsu, H.; Rovis, T. Angew. Chem. Int. Ed. 2019, 58, 4002. (o) Guin, S.; Dolui, P.; Zhang, X.; Paul, S.; Singh, V. K.; Pradhan, 
S.; Chandrashekar, H. B.; Anjana, S. S.; Paton, R. S.; Maiti, D. Angew. Chem. Int. Ed. 2019, 58, 5633. (p) Whitehurst, W. G.; Blackwell, J. H.; Hermann, G. N.; Gaunt, M. J. Angew. Chem. Int. Ed. 2019, 58, 9054. (q) Ma, Y.; Yao, X.; Zhang, L.; Ni, P.; Cheng, R.; Ye, J. Angew. Chem. Int. Ed. 2019, 58, 16548. (r) Grainger, R.; Heightman, T. D.; Ley, S. V.; Lima, F.; Johnson, C. N. Chem. Sci. 2019, 10, 2264. (s) Vasu, D.; Fuentes de Arriba, A. L.; Leitch, J. A.; de Gombert, A.; Dixon, D. J. Chem. Sci. 2019, 10, 3401. (t) Asako, S.; Ishihara, S.; Hirata, K.; Takai, K. J. Am. Chem. Soc. 2019, 141, 9832. (u) Lin, W.; Zhang, K.-F.; Baudoin, O. Nat. Catal. 2019, 2, 882. (v) Chan, J. Z.; Chang, Y.; Wasa, M. Org. Lett. 2019, 21, 984. (w) Zhou, L.; Shen, Y.-B.; An, X.-D.; Li, X.-J.; Li, S.-S.; Liu, Q.; Xiao, J. Org. Lett. 2019, 21, 8543. (x) Kataoka, M.; Otawa, Y.; Ido, N.; Mori, K. Org. Lett. 2019, 21, 9334. (y) Lee, M.; Adams, A.; Cox, P. B.; Sanford, M. S. Synlett 2019, 30, 417. (z) Kapoor, M.; ChandThakuri, P.; Maxwell, J. M.; Liu, D.; Zhou, H.; Young, M. C. Synlett 2019, 30, 519. (aa) Ohmatsu, K.; Suzuki, R.; Furukawa, Y.; Sato, M.; Ooi, T. ACS Catal. 2020, 10, 2627. (ab) Roque, J. B.; Kuroda, Y.; Jurczyk, J.; Xu, L.-P.; Ham, J. S.; Göttemann, L. T.; Roberts, C. A.; Adpressa, D.; Saurí, J.; Joyce, L. A.; Musaev, D. G.; Yeung, C. S.; Sarpong, R. ACS Catal. 2020, 10, 2929. (ac) Rand, A. W.; Yin, H.; Xu, L.; Giacoboni, J.; Martin-Montero, R.; Romano, C.; Montgomery, J.; Martin, R. ACS Catal. 2020, 10, 4671. (ad) Liu, W.; Babl, T.; Röther, A.; Reiser, O.; Davies, H. M. L. Chem. Eur. J. 2020, 26, 4236. (ae) Verma, P.; Richter, J. M.; Chekshin, N.; Qiao, J. X.; Yu, J.-Q. J. Am. Chem. Soc. 2020, 142, 5117. (af) Walker, M. M.; Koronkiewicz, B.; Chen, S.; Houk, K. N.; Mayer, J. M.; Ellman, J. A. J. Am. Chem. Soc. 2020, 142, 8194. (ag) Feng, K.; Quevedo, R. E.; Kohrt, J. T.; Oderinde, M. S.; Reilly, U.; White, M. C. Nature 2020, 580, 621. (ah) Sarver, P. J.; Bacauanu, V.; Schultz, D. M.; DiRocco, D. A.; Lam, Y.-h.; Sherer, E. C.; MacMillan, D. W. C. Nat. Chem. 2020, 12, 459. (ai) McManus, J. B.; Onuska, N. P. R.; Jeffreys, M. S.; Goodwin, N. C.; Nicewicz, D. A. Org. Lett. 2020, 22, 679. (aj) Oeschger, R.; Su, B.; Yu, I.; Ehinger, C.; Romero, E.; He, S.; Hartwig, J. Science 2020, 368, 736. (ak) Short, M. A.; Blackburn, J. M.; Roizen, J. L. Synlett 2020, 31, 102.

(3) (a) Zhang, C.; De C, K.; Mal, R.; Seidel, D. J. Am. Chem. Soc. 2008, 130, 416. (b) Zheng, L.; Yang, F.; Dang, Q.; Bai, X. Org. Lett. 2008, 10, 889. (c) Dieckmann, A.; Richers, M. T.; Platonova, A. Y.; Zhang, C.; Seidel, D.; Houk, K. N. J. Org. Chem. 2013, 78, 4132. (d) Richers, M. T.; Deb, I.; Platonova, A. Y.; Zhang, C.; Seidel, D. Synthesis 2013, 45, 1730.

(4) (a) Richers, M. T.; Breugst, M.; Platonova, A. Y.; Ullrich, A.; Dieckmann, A.; Houk, K. N.; Seidel, D. J. Am. Chem. Soc. 2014, 136, 6123. (b) Jarvis, C. L.; Richers, M. T.; Breugst, M.; Houk, K. N.; Seidel, D. Org. Lett. 2014, 16, 3556. (c) Mahato, S.; Haque, M. A.; Dwari, S.; Jana, C. K. RSC Adv. 2014, 4, 46214.

(5) (a) Ma, L.; Seidel, D. Chem. Eur. J. 2015, 21, 12908. (b) Paul, A.; Chandak, H. S.; Ma, L.; Seidel, D. Org. Lett. 2020, 22, 976.

(6) (a) Li, J.; Qin, C.; Yu, Y.; Fan, H.; Fu, Y.; Li, H.; Wang, W. Adv. Synth. Catal. 2017, 359, 2191. (b) Li, J.; Fu, Y.; Qin, C.; Yu, Y.; Li, H.; Wang, W. Org. Biomol. Chem. 2017, 15, 6474. (c) Zhu, Z.; Seidel, D. Org. Lett. 2017, 19, 2841.

(7) Paul, A.; Adili, A.; Seidel, D. Org. Lett. 2019, 21, 1845.

(8) Additional examples of amine redox-annulations: (a) Zhang, C.; Das, D.; Seidel, D. Chem. Sci. 2011, 2, 233. (b) Kang, Y.; Chen, W.; Breugst, M.; Seidel, D. J. Org. Chem. 2015, 80, 9628. (c) Chen, W.; Seidel, D. Org. Lett. 2016, 18, 1024. (d) Zhu, Z.; Lv, X.; Anesini, J. E.; Seidel, D. Org. Lett. 2017, 19, 6424. (e) Zhu, Z.; Chandak, H. S.; Seidel, D. Org. Lett. 2018, 20, 4090. (f) Liu, Y.; Wu, J.; Jin, Z.; Jiang, H. Synlett 2018, 29, 1061.
(9) For detailed discussions on the mechanisms of these transformations, see references: (a) Xue, X.; Yu, A.; Cai, Y.; Cheng, J.-P. Org. Lett. 2011, 13, 6054. (b) Ma, L.; Paul, A.; Breugst, M.; Seidel, D. Chem. Eur. J. 2016, 22, 18179; see also refs 1m, 3c, 4a, 4b, and 8 b.

(10) Examples of redox-neutral $\alpha-\mathrm{C}-\mathrm{H}$ bond annulations of secondary amines that likely involve a pericyclic step: (a) Grigg, R.; Nimal Gunaratne, H. Q.; Henderson, D.; Sridharan, V. Tetrahedron 1990, 46, 1599. (b) Soeder, R. W.; Bowers, K.; Pegram, L. D.; Cartaya-Marin, C. P. Synth. Commun. 1992, 22, 2737. (c) Grigg, R.; Kennewell, P.; Savic, V.; Sridharan, V. Tetrahedron 1992, 48, 10423. (d) Deb, I.; Seidel, D. Tetrahedron Lett. 2010, 51, 2945. (e) Kang, Y.; Richers, M. T.; Sawicki, C. H.; Seidel, D. Chem. Commun. 2015, 51, 10648. (f) Cheng, Y.-F.; Rong, H.-J.; Yi, C.-B.; Yao, J.-J.; Qu, J. Org. Lett. 2015, 17, 4758. (g) Yang, Z.; Lu, N.; Wei, Z.; Cao, J.; Liang, D.; Duan, H.; Lin, Y. J. Org. Chem. 2016, 81, 11950. (h) Rong, H.-J.; Cheng, Y.-F.; Liu, F.-F.; Ren, S.-J.; Qu, J. J. Org. Chem. 2017, 82, 532. (i) Purkait, A.; Roy, S. K.; Srivastava, H. K.; Jana, C. K. Org. Lett. 2017, 19, 2540.

(11) (a) Chrzanowska, M.; Rozwadowska, M. D. Chem. Rev. 2004, 104 3341. (b) Grycova, L.; Dostal, J.; Marek, R. Phytochemistry 2007, 68, 150. (c) Bhadra, K.; Kumar, G. S. Med. Res. Rev. 2011, 31, 821. (d) Yu, J.; Zhang, Z.; Zhou, S.; Zhang, W.; Tong, R. Org. Chem. Front. 2018, 5, 242.

(12) (a) Enders, D.; Wang, C.; Bats, J. W. Synlett 2009, 1777. (b) Enders, D.; Hahn, R.; Atodiresei, I. Adv. Synth. Catal. 2013, 355, 1126. (c) Hahn, R.; Jafari, E.; Raabe, G.; Enders, D. Synthesis 2015, 47, 472 .

(13) Fessard, T. C.; Motoyoshi, H.; Carreira, E. M. Angew. Chem. Int. Ed. 2007, 46, 2078.

(14) Ono, N.; Miyake, H.; Kamimura, A.; Hamamoto, I.; Tamura, R.; Kaji, A. Tetrahedron 1985, 41, 4013.

(15) Decarboxylative annulations: (a) Cohen, N.; Blount, J. F.; Lopresti, R. J.; Trullinger, D. P. J. Org. Chem. 1979, 44, 4005. (b) Tang, M.; Tong, L.; Ju, L.; Zhai, W.; Hu, Y.; Yu, X. Org. Lett. 2015, 17, 5180. (c) Kang, Y.; Seidel, D. Org. Lett. 2016, 18, 4277. (d) Wu, J.-s.; Jiang, H.-j.; Yang, J.-g.; Jin, Z.-n.; Chen, D.-b. Tetrahedron Lett. 2017, 58, 546. (e) Paul, A.; Thimmegowda, N. R.; Galani Cruz, T.; Seidel, D. Org. Lett. 2018, 20, 602; see also references 3a, 3b, 3d, 5b, 6c, 8a, 8e, and 14 ..

(16) Selected reviews on decarboxylative coupling reactions not limited to amino acids: (a) Rodriguez, N.; Goossen, L. J. Chem. Soc. Rev. 2011, 40, 5030. (b) Xuan, J.; Zhang, Z.-G.; Xiao, W.-J. Angew. Chem. Int. Ed. 2015, 54, 15632. (c) Patra, T.; Maiti, D. Chem. Eur. J. 2017, 23, 7382. (d) Wei, Y.; Hu, P.; Zhang, M.; Su, W. Chem. Rev. 2017, 117, 8864. (e) Rahman, M.; Mukherjee, A.; Kovalev, I. S.; Kopchuk, D. S.; Zyryanov, G. V.; Tsurkan, M. V.; Majee, A.; Ranu, B. C.; Charushin, V. N.; Chupakhin, O. N.; Santra, S. Adv. Synth. Catal. 2019, 361, 2161.

(17) (a) Kind, T.; Fiehn, O. BMC Bioinformatics 2007, 8, 105. (b) Wang, Y.; Gu, M. Anal. Chem. 2010, 82, 7055.

(18) Starting material synthesis: (a) Ghislieri, D.; Green, A. P.; Pontini, M.; Willies, S. C.; Rowles, I.; Frank, A.; Grogan, G.; Turner, N. J. J. Am. Chem. Soc. 2013, 135, 10863. (b) Gray, N. M.; Cheng, B. K.; Mick, S. J.; Lair, C. M.; Contreras, P. C. J. Med. Chem. 1989, 32, 1242. (c) Ji, Y.; Wang, J.; Chen, M.; Shi, L.; Zhou, Y. Chin. J. Chem. 2018, 36, 139. (d) Tamayo, N. A.; Bo, Y.; Gore, V.; Ma, V.; Nishimura, N.; Tang, P.; Deng, H.; Klionsky, L.; Lehto, S. G.; Wang, W.; Youngblood, B.; Chen, J.; Correll, T. L.; Bartberger, M. D.; Gavva, N. R.; Norman, M. H. J. Med. Chem. 2012, 55, 1593. (e) Ji, Y.; Shi, L.; Chen, M.-W.; Feng, G.-S.; Zhou, Y.-G. J. Am. Chem. Soc. 2015, 137, 10496. (f) Zhao, Z.; Sun, Y.; Wang, L.; Chen, X.; Sun, Y.; Lin, L.; Tang, Y.; Li, F.; Chen, D. Tetrahedron 
Lett. 2019, 60, 800. (g) Schönbauer, D.; Sambiagio, C.; Noël, T.; Schnürch, M. Beilstein J. Org. Chem. 2020, 16, 809. (h) Cutter, P. S.; Miller, R.; Schore, N. E. Tetrahedron 2002, 58, 1471. (i) Bailey, D. M.; Degrazia, C. G.; Lape, H. E.; Frering, R.; Fort, D.; Skulan, T. J. Med. Chem. 1973, 16, 151. (j) See also ref 12b.
(19) (a) Kraus, G. A.; Wu, T. A. Tetrahedron 2010, 66, 569. (b) Dai-Ho, G.; Mariano, P. S. J. Org. Chem. 1988, 53, 5113. (c) Orito, K.; Satoh, Y.; Nishizawa, H.; Harada, R.; Tokuda, M. Org. Lett. 2000, 2, 2535. (d) Azzena, U. J. Chem. Soc., Perkin Trans. 1 2002, 360. (e) Lahm, G.; Stoye, A.; Opatz, T. J. Org. Chem. 2012, 77, 6620. 